\title{
Gestión de las organizaciones cooperativas: un análisis desde la teoría de la
} agencia.

Management of cooperative organizations: an analysis from the theory of the agency.

\section{Joana Rivas de Alexis ${ }^{1}$ y Víctor J. Alexis ${ }^{2}$}

1. Centro Regional Universitario de Colón. Especialista en Gerencia General.

Estudiante de Maestría en Universidad del Istmo. Email: joanamil100@gmail.com. https://orcid.org/0000-0002-6254-9985

2. Profesor Titular de Economía. Centro Regional Universitario de Colón, Universidad de Panamá. Email: victor.alexis@up.ac.pa., javieralexis25@yahho.com. https://orcid.org/0000-0003-3147-6565

Págs.: 56 - 69

\section{Recibido: 12 /8/2020}

Aprobado: 18/8/2020

\section{Resumen}

La importancia y reconocimiento que vienen teniendo las cooperativas, por su potencial para generar oportunidades de desarrollo humano, justifica los estudios que desde distintos ámbitos se puedan realizar sobre éstas, a fin de profundizar su comprensión como fenómenos sociales y organizacionales. En este artículo se presenta un análisis de las cooperativas desde la óptica de la teoría de la agencia, enfoque que aporta valor conceptual y metodológico para la investigación y gestión de las organizaciones. Se sostiene que dada las características, principios y valores en que se fundamentan las cooperativas, estos no favorecen o estimulan el surgimiento de problemas de agencia; lo que contribuye al gerenciamiento de estas organizaciones para potenciar su capacidad de aporte a la consecución de los Objetivos de Desarrollo Sostenible.

Palabras clave: cooperativas, economía social, teoría de la agencia, nueva economía institucional. 


\begin{abstract}
The importance and recognition that cooperatives have been having, because of their potential to generate opportunities for human development, justifies the studies that can be carried out from different fields on them, in order to deepen their understanding as social and organizational phenomena. This article presents an analysis of cooperatives from the perspective of agency theory, an approach that provides conceptual and methodological value for the research and management of organizations. It is argued that given the characteristics, principles and values on which cooperatives are based, they do not favor or stimulate the emergence of agency problems; which contributes to the management of these organizations to enhance their capacity to contribute to the achievement of the Sustainable Development Goals.
\end{abstract}

Keywords: cooperatives, social economy, agency theory, new institutional economy.

\title{
Introducción
}

A nivel mundial y nacional se viene reconociendo a las organizaciones o empresas cooperativas como alternativas para generar oportunidades de generación de desarrollo humano, sobre todo a partir del debate en torno a la globalización neoliberal. Es así que la Organización de las Naciones Unidas para la Agricultura y la Alimentación, citada por Campos (2019), estableció que: “La gran ambición de los Objetivos de Desarrollo Sostenible (ODS) puede solamente ser alcanzada a través de la cooperación y las alianzas mundiales, entre múltiples actores y en un amplio rango de áreas, siendo las cooperativas parte de ellas" (p.13).

En la Resolución aprobada por la Asamblea General de las Naciones Unidas del 18 de diciembre de 2019, ONU (2019), se establece que:

Las cooperativas, en sus distintas formas, promueven la máxima participación posible en el desarrollo económico y social de las comunidades locales y de todas las personas, incluidas las mujeres, la juventud, las personas de edad, las personas con discapacidad y los pueblos indígenas, cuya inclusión refuerza el desarrollo económico y social, y contribuyen a la erradicación de la pobreza y el hambre. 
En Panamá, esa apuesta por el movimiento cooperativo para hacer frente a la pobreza queda establecido en el actual Plan Estratégico del Gobierno Nacional 20202024, el cual reconoce en el cooperativismo, (PEG, 2020) "un sistema organizacional importante de colaboración económica y participación en el desarrollo del país" (p. 110). Igualmente, el Plan Estratégico Institucional 2020-2024 del Instituto Panameño Autónomo Cooperativo (IPACOOP), presenta dentro de sus Ejes Estratégicos, fomentar y consolidar las cooperativas como un factor clave para el desarrollo socioeconómico del país; y promover y dinamizar las cooperativas en los sectores con pobreza multidimensional.

Lo anterior evidencia el reconocimiento e importancia que están teniendo las cooperativas. Esto, precisamente, justifica los estudios que desde distintos ámbitos se puedan realizar de éstas, para profundizar su comprensión como fenómenos sociales y organizacionales. Es así que en este artículo se presenta un análisis de las cooperativas desde la óptica de teoría de la agencia, enfoque del cuerpo teórico de la Nueva Economía Institucional, que aporta valor conceptual y metodológico para la investigación y gestión de las organizaciones; entendiendo las organizaciones como sistemas complejos y dinámicos que se desenvuelven en el marco de reglas (instituciones) que establecen sus límites e incentivos y, además, son las organizaciones mecanismos mediante la cual se generan los bienes y servicios que las sociedades demandan para satisfacer las necesidades humanas (Alexis, 2020).

El objetivo de analizar desde la teoría de la agencia las organizaciones cooperativas, apunta a sostener que dada las características, principios y valores en que se fundamentan las cooperativas, estos no favorecen o estimulan conductas relacionadas a problemas de agencia; lo que contribuye al gerenciamiento de estas organizaciones para potenciar su capacidad de aporte a la consecución de los Objetivos de Desarrollo Sostenible. Para este objetivo, se realizó una indagación documental aplicando criterios de búsqueda relacionados con cooperativismo, economía social, teoría de la agencia y nueva economía institucional, entre otros; seleccionando contenidos cuyos conceptos, teorías y perspectivas resultaron útiles para el análisis y conclusiones enfocadas al objetivo de esta publicación. 


\section{Materiales y Métodos}

\section{Concepto y características de las cooperativas como organización.}

Desde la primera aparición reconocida oficialmente de una organización cooperativa de Consumo en 1844, en Rochdale, Inglaterra, las cooperativas han ido ganando reconocimiento a lo largo de su evolución histórica, por sus características organizacionales en términos de sus principios y valores que la sustentan.

La Alianza Internacional de Cooperativas (ACI), en su declaración sobre Identidad y Principios Cooperativos, define la Cooperativa como: una asociación autónoma de personas unidas voluntariamente para hacer frente a sus necesidades y aspiraciones comunes en materia económica, social y cultural mediante una empresa de propiedad conjunta y de gestión conjunta.

De acuerdo con Schwettmann (s/f):

Por su propia naturaleza, las cooperativas desempeñan un triple papel: como actores económicos crean oportunidades de empleo, medios de vida e ingresos; Como las organizaciones sociales se basan en un objetivo común y un vínculo común, extienden la protección y la seguridad, y contribuyen a la igualdad y la justicia social; y como asociaciones de individuos controladas democráticamente, desempeñan un papel constructivo en las comunidades y las naciones, en la sociedad y la política. Estos tres roles no pueden disociarse uno del otro porque son inherentes a la naturaleza misma de las cooperativas. (p.2).

El Cooperativismo se fundamenta en un conjunto de valores y principios que le dan su identidad, tal como se presenta en la siguiente Tabla 1. 
Tabla 1.

Principios y valores del cooperativismo.

\begin{tabular}{ll}
\hline \multicolumn{1}{c}{ Principios } & \multicolumn{1}{c}{ Valores } \\
\hline Asociación Voluntaria y Abierta & Igualdad, Equidad \\
Administración Democrática por los Asociados & Democracia \\
Participación Económica de los Asociados & Justicia Económica \\
Autonomía e Independencia & Autoayuda, Democracia \\
Educación, Formación e Información & Auto responsabilidad \\
Cooperación entre Cooperativas. & Autoayuda \\
Interés por la Comunidad. & Solidaridad
\end{tabular}

Nota: Elaborado en base a la Alianza Internacional de Cooperativas (ACI)

Las organizaciones cooperativas, sustentadas en el trabajo y la solidaridad, constituye un modelo organizativo representativo de la Economía Social, que la diferencia de la empresa típicamente capitalista cuyo objetivo es el lucro; mientas, las cooperativas son organizaciones cuyo objetivo es el bien común. En ese sentido, cabe destacar el planteamiento de Walras, quien al referirse a las cooperativas señaló que “...cumplen su gran papel económico que es no suprimir el capital sino hacer que el mundo sea menos capitalista, y también un papel moral, no menos considerable, que consiste en introducir la democracia en el mecanismo de la producción" (Walras, 1989, como se citó en Chaves, Monzón y Sajardo, 2003, p. 46). En la siguiente tabla 2 se puede observar estas diferencias. 
Tabla 2.

Diferencias entre empresas capitalistas y cooperativas.

\begin{tabular}{|c|c|c|c|}
\hline Elementos & & Capitalistas & Cooperativas \\
\hline Titulares & & Accionistas - socios & Asociados \\
\hline Objetivo & & $\begin{array}{l}\text { Maximizar beneficios en relación } \\
\text { al capital aportado }\end{array}$ & $\begin{array}{l}\text { Brindar servicios y/o } \\
\text { productos a sus asociados } \\
\text { sin discriminación alguna }\end{array}$ \\
\hline Control y dirección & & En función del capital invertidos & $\begin{array}{l}\text { En función de los } \\
\text { Asociados (un hombre un } \\
\text { voto) }\end{array}$ \\
\hline Poder de decisión & & $\begin{array}{l}\text { A cargo de los propietarios y en } \\
\text { relación al capital integrado. }\end{array}$ & $\begin{array}{l}\text { Se basa en el principio de } \\
\text { igualdad, Todos los } \\
\text { asociados poseen los } \\
\text { mismos derechos a opinar y } \\
\text { votar }\end{array}$ \\
\hline Capital & & $\begin{array}{l}\text { Fijo, por lo tanto, generalmente } \\
\text { no puede disminuir del monto } \\
\text { constituido }\end{array}$ & $\begin{array}{l}\text { Variable, de acuerdo al } \\
\text { ingreso o egreso de los } \\
\text { asociados, }\end{array}$ \\
\hline $\begin{array}{l}\text { Condiciones } \\
\text { ingreso }\end{array}$ & de & $\begin{array}{l}\text { Se invierte y se reparte entre sus } \\
\text { accionistas en proporción al } \\
\text { capital invertido }\end{array}$ & $\begin{array}{l}\text { Libre, sujeta a aprobación } \\
\text { del resto de los asociados }\end{array}$ \\
\hline $\begin{array}{l}\text { Ganancia } \\
\text { excedentes }\end{array}$ & o & $\begin{array}{l}\text { Se invierte y se reparte entre sus } \\
\text { accionistas en proporción al } \\
\text { capital invertido }\end{array}$ & $\begin{array}{l}\text { Se capitaliza o se distribuye } \\
\text { entre los asociados en } \\
\text { proporción } \\
\text { operaciones }\end{array}$ \\
\hline
\end{tabular}

Nota: Tomado de Izquierdo (2005).

Estas características de las cooperativas le posibilitan unos roles diferentes a los de las empresas típicamente capitalistas, les da valores agregados a las cooperativas para impactos sociales y económicos específicos. 


\section{La Teoría de la Agencia.}

La Teoría de la Agencia se inscribe dentro del cuerpo teórico de la Nueva Economía Institucional (NEI), enfoque de la economía que tiene su origen en los trabajos de Ronald Coase con su ensayo "The Nature of the Firm" publicado en $1937 \mathrm{y}$ los posteriores aportes de Douglas North, Oliver Williamson y otros. La NEI destaca la importancia y papel de las instituciones en las organizaciones, en el crecimiento y desarrollo de los países; y, representa una importante contribución conceptual y metodológica para la investigación y gestión de las organizaciones.

Para los propósitos de nuestro análisis, es importante destacar dos conceptos básicos en la NEI, estos son los conceptos de Instituciones y Organizaciones.

Las Instituciones, North (1993) las define como "las reglas del juego en una sociedad o, más formalmente, las limitaciones ideadas por el hombre que dan forma a la interacción humana. Por consiguiente, estructuran incentivos en el intercambio humano, sea político, social o económico" (p. 13). Mientras, las Organizaciones son los jugadores, grupos de individuos unidos por un objetivo común.

Las instituciones pueden ser formales o informales. Las informales son las sanciones, tabús, costumbres, tradiciones y códigos de conducta; y las formales son las constituciones, las leyes y los derechos de propiedad (North, 1993).

Por su parte, Rivas (2003) al referirse a la importancia de las instituciones en el neoinstitucionalismo económico indica que:

Son importantes como elementos fundamentales del contexto estratégico, dado que imponen una serie de restricciones al comportamiento basado en el interés personal, es decir, definen o restringen las estrategias que los actores políticos, sociales y económicos adoptan en la lucha y en los procesos por alcanzar sus objetivos. (p.42)

La diferencia entre Instituciones y Organizaciones puede radicar en que “mientras las primeras son un grupo de reglas que estructuran la interacción entre actores, las segundas son actores colectivos que pueden ser sujetos a estas restricciones institucionales” (Knight, 2004, como se citó en Briseño Ramírez y Sánchez, 2019, 
p.8). Una institución toma vida dentro de un actor o un conjunto de actores, de una organización; y una organización necesita reglas para poder llevar a cabo sus metas.

La Teoría de la Agencia, fue formalizada por los economistas Jensen y Meckling en 1976. Para Jensen y Meckling, citados por Nunes (2018), la organización se concibe como:

Una red de contratos, ya sean ellos explícitos o implícitos, los cuales establecen las funciones y definen los derechos y los deberes de todos sus stakeholders, y cuyo objetivo es maximizar su función de utilidad particular y ser capaces de prever los elementos del contrato sobre el valor futuro de su patrimonio. De esas relaciones surgen las figuras del "Principal" y del “Agente". (párr.1)

Las Relaciones de Agencia constituyen, y se entiende, como un contrato donde una o más personas, denominados Principal, solicitan los servicios de otra persona, denominada Agente, para que este realice en su nombre una determinada tarea, lo que implica delegarle de capacidad para tomar decisiones. Por lo tanto, una Relación de Agencia se da cuando, bajo un contrato, el Principal delega al Agente autoridad para tomar decisiones con el fin de desempeñar alguna actividad o servicio en su nombre.

La teoría se basa en el análisis de los conflictos que puede producirse entre el llamado Principal (Propietario) y el Agente (Gerente), pues ambas figuras tratan de maximizar su actividad propia, produciéndose eventualmente conflictos de intereses.

Los dueños de una organización (el Principal) al delegar responsabilidades a sus subordinados (Agentes), éstos, con la intención de satisfacer objetivos personales, pueden no tomar las decisiones que maximicen el beneficio de los propietarios, tomando ventaja de su información privilegiada. Es ahí cuando se dice que existen Problemas de Agencia. El problema agente-principal se da, por tanto, cuando los Agentes buscan su beneficio personal dejando de lado los objetivos de los dueños o principales. Cuando surgen estos problemas aparecen los Costes de Agencia.

La teoría asume que existe información asimétrica entre el Agente y el Principal, y diferentes niveles de aversión al riesgo. La asimetría en la información se refiere a que el dueño o Principal no sabe todo lo que el Agente realiza en cada momento, y si está actuando en concordancia con los objetivos planteados. Esta asimetría de información, junto con el oportunismo, que Williamson define como la búsqueda del interés propio 
con dolo, permite identificar dos aspectos de un Problema de Agencia: el Riesgo Moral y la Selección Adversa.

El Riesgo Moral es un concepto económico que dice cómo los individuos asumen riesgos cuando los resultados negativos de estas decisiones no la asumen ellos, sino otro. Es un tipo de comportamiento oportunista donde, valiéndose de información asimétrica, el Agente asume conductas o acciones cuyos costos, como resultado de las consecuencias negativas que puedan acarrear estas acciones, son soportados por el Principal.

La Selección Adversa, a su vez, se refiere a la falta de competencia o capacidad del Agente para realizar las actividades que le designa el Principal; es decir, el Agente presume tener habilidades que no posee cuando fue contratado.

La eficiencia en las Relaciones de Agencia significa para la nueva economía institucional obtener bajos costos de transacción erradicando incertidumbres y maximizando la capacidad de monitorear y controlar las transacciones (Parsons, 2007).

La esencia de un Problema de Agencia, según Clarke (2004), es la separación de la propiedad de una organización de su control. En otras palabras, la separación de quienes dan los recursos financieros o son titulares de los derechos de propiedad, y de quienes tienen el control (el gerente).

Conviene que el Principal intente anular o minimizar las divergencias generadas por la información asimétrica estableciendo incentivos para el Agente y limitando su actuación. Para lograrlo, necesita diseñar instituciones que generen incentivos para que cuando el Agente busque sus propios intereses esté buscando los del principal (Caballero, 2011). Estos incentivos y limitaciones son lo que llaman Costos de Agencia. Estos costos se identifican como: - Coste de Negociación: soportado por ambas partes; Coste de vigilancia: soportados por el Principal y destinados a evitar el comportamiento oportunista del Agente; Coste de Garantía: soportados por el Agente y destinado a mitigar la desconfianza del Principal y, Coste de oportunidad: serían las pérdidas que soporta el Principal, debido a la divergencia de intereses existentes entre él y el Agente. 


\section{Resultados y Discusión}

\section{El gerenciamiento de las cooperativas desde la teoría de la agencia.}

Esta perspectiva de la nueva economía institucional, en la teoría de la agencia, constituye un fundamento importante de la viabilidad gerencial de las cooperativas, dado que los principios y valores del cooperativismo, entendiéndolos como instituciones de este tipo de organizaciones de economía social, no favorecen la aparición de problemas de agencia, esto es, oportunismo; lo que contribuye a ser organizaciones con potencial generador de oportunidades para el desarrollo humano. En ese sentido, para respaldar este planteamiento central del análisis, se hace referencia a las siguientes fuentes:

En primer lugar, cabe destacar el artículo de investigación titulado Antecedentes del oportunismo en las cooperativas agroalimentarias. El objetivo de este trabajo fue conocer las causas del oportunismo en las cooperativas agroalimentarias. La población objeto de estudio estuvo integrada por las cooperativas agroalimentarias españolas de primer grado. En atención a los resultados de la investigación, los autores de esta publicación, Sánchez-Navarro, Arcas-Lario, y Hernández-Espallardo (2019), concluyen que: se ha encontrado evidencia empírica del efecto negativo que sobre el oportunismo de los socios tiene la dependencia que tienen de la cooperativa y de la orientación a largo plazo de la relación que mantienen con ella. Conforme los socios son más dependientes de la cooperativa, en mayor medida la necesitan para conseguir sus objetivos $\mathrm{y}$, por lo tanto, cabe pensar que estos evitarán comportarse de forma oportunista por miedo a las repercusiones que ello podría tener, incluida su expulsión. (p.129)

Las conclusiones de Sánchez-Navarro y otros (2019) en su estudio sobre el oportunismo, uno de los más importantes que se estudian en la Teoría de la Agencia, en cooperativas agroalimentarias, lleva a plantear que al existir factores como homogeneidad, dependencia y orientación hacia los socios en las cooperativas, estos factores no favorecen la aparición de los llamados problemas de agencia, favoreciendo el gerenciamiento enfocado a los objetivos de la organización. 
En segundo lugar, las cooperativas pertenecen a la economía social, donde una característica distintiva de las organizaciones de la economía social es el carácter democrático en su gestión. Como señala Chávez y Monzón (2018):

El criterio democrático se considera esencial para considerar a una empresa parte de la economía social, puesto que la utilidad social de estas empresas se basa en su propósito social y en los valores democráticos y participativos que aportan a la gestión de la empresa. (p.15)

Estos autores señalan que la caracterización de las formas organizativas se realiza atendiendo a tres criterios: el objetivo de la organización, el modo de decisión y el modo de distribución de los beneficios y excedentes. Para el caso de la economía social, la definición responde a estos tres criterios: son empresas cuya finalidad es dar servicio a los socios y/o a la colectividad, su proceso decisional asegura la participación equilibrada de socios y otros agentes interesados en el objeto de la entidad sin que en ningún caso prevalezca el interés del inversor capitalista, y finalmente su modo de distribución de beneficios y excedentes, si los hay, prioriza a las personas y al factor trabajo frente al capital (Chávez y Monzón, 2018).

Esta definición para el caso de las empresas de la economía social, en la cual se inscriben las cooperativas, lleva a señalar desde la teoría de agencia de que, dada las características e instituciones de las cooperativas, esto es, sus principios y valores organizacionales, como parte de sus reglas de juego, de igual manera no favorece o estimula el surgimiento de problemas de agencia.

En tercer lugar, la ACI hace referencia al hecho de que las cooperativas enseñan buenas prácticas democráticas, ayudan a construir solidaridad y cohesión en las comunidades locales, desarrollan la capacidad de liderazgo y apoyan la capacitación y la educación. Además, se concentran en las necesidades humanas y no en su codicia, mediante empresas que pertenecen a sus integrantes y que permiten el desarrollo de su propia comunidad. Al redistribuir las ganancias entre ellos, mantienen la riqueza y pueden seguir creciendo. De esa forma, señala la ACI, las cooperativas reducen los conflictos; lo que precisamente evidencia que estas características de las cooperativas son contrarias a favorecer en este tipo de organizaciones las conductas oportunistas, como problema que puede surgir en una relación de agencia. 


\section{Conclusiones}

La NEI explica el funcionamiento de las organizaciones desde el enfoque de lo contractual; es decir, bajo la figura del contrato, donde se establece las reglas de juego (instituciones) entre el Agente y el Principal. De este cuerpo teórico se entiende que en la medida en que las instituciones, las reglas de juego, sean transparentes, generen confianza y certidumbre, ello reduce los costos de agencia.

Las cooperativas poseen características distintivas como organizaciones, que la identifican con la economía social, al basarse en una estructura de propiedad diferente, con lógica de funcionamiento y objetivos distintos a la empresa típicamente capitalista. Estas características influyen en la toma de decisiones gerenciales, a fin de maximizar los resultados de la gestión en favor de los socios cooperativistas.

Las cooperativas como organizaciones de economía social, resultan viable desde el punto de vista gerencial, analizado desde la teoría de la agencia, ya que al existir homogeneidad, dependencia y orientación hacia los socios, estos factores no favorecen o estimulan el surgimiento y desarrollo de problemas de agencia, como el oportunismo, lo que se evidencia en los resultados de la investigación de Sánchez-Navarro, ArcasLario, y Hernández-Espallardo. Además, los principios y valores cooperativos como instituciones del cooperativismo, de igual manera operan en desfavor de las conductas oportunistas. Esto crea condiciones para que el gerenciamiento de estas organizaciones sea más exitoso en términos del logro de los objetivos organizacionales que se propongan los socios cooperativistas.

Lo anterior también permite concluir que las organizaciones cooperativas son un modelo alternativo para generar oportunidades de desarrollo humano, en términos de empleo e ingresos; sobre todo en las actuales circunstancias donde la pandemia Covid19 representa un revés para el logro de los ODS por la pérdida de empleos y, en consecuencia, el aumento de la pobreza a nivel mundial. 


\section{Referencias Bibliográficas}

Alexis, V. J (2020). Las organizaciones de economía solidaria como modelo alternativo para la generación de oportunidades de desarrollo humano en Panamá. Tesis de doctorado. Universidad del Caribe, Panamá.

Briseño Ramírez, H. y Sánchez Bernal, A. (2019). Reflexiones en torno a la nueva economía institucional y el desempeño de las organizaciones públicas. Cooperativismo \& Desarrollo, 27(2), 1-23. doi: https://doi.org/10.16925/23824220.2019.02.09

Caballero, G (2011). Perspectivas de análisis institucional contemporáneo: enfoques, métodos y experimentos. Ekonomiaz, 77(2), 222-251. file:///C:/Users/p.indesco.nal/ Downloads/9.pdf.

Campos M, J. (2019). Cooperativismo: una organización idónea para impulsar los Objetivos de Desarrollo Sostenible (ODS)-2030 establecidos por la Organización de las Naciones Unidas (ONU). Universidad FUNDEPOS. https://www.cene.coop/wp-content/uploads/2019/10/Cooperativas-y_los_ODS2030.pdf

Chaves, R. \& Monzón, J.L. (2018): “La economía social ante los paradigmas económicos emergentes: innovación social, economía colaborativa, economía circular, responsabilidad social empresarial, economía del bien común, empresa social y economía solidaria”, CIRIEC España, Revista de Economía Pública, Social y Cooperativa, 93, 5-50, DOI: 10.7203/CIRIEC-E.93.12901.

Chaves, R., Monzón, J.L., y Sajardo, A (2003). Elementos de economía social. Teoría y Realidad. Universidad de Valencia.

Clarke, T. (2004). Introduction: Theories of Governance - Reconceptualizing. Corporate governance Theory After the Enron Experience. In T. Clarke. (Ed.). Theories of Corporate Governance. The Philosophical Foundations of Corporate Governance. USA and Canada: Routledge.

Izquierdo A., C. (2005). El cooperativismo una alternativa de desarrollo a la globalización neoliberal para América Latina. www.eumed.net/libros/2005/ceia/ 
North, D. (1993). Instituciones, cambio institucional y desempeño económico. Chile: FCE. https://www.buscalibre.cl/libro-instituciones-cambio-institucional-ydesempeno-economico/9786071619525/p/46694006

Nunes, P. (2018). Teoría de la Agencia. Recuperado el 8 de agosto de https://knoow.net/es/cieeconcom/finanzas/teoria-la-agencia/

ONU. Resolución aprobada por la Asamblea General el 19 de diciembre de 2019. https://base.socioeco.org/docs/a_res_74_119-es.pdf

Parsons, W. (2007). Políticas Públicas. Una introducción a la teoría y a la práctica del análisis de las políticas públicas. México: FLACSO. https://campusvirtual.univalle.edu.co/moodle/pluginfile.php/1097951/mod_re source/content/1/Parsons\%20Wayne\%20-\%20 Politicas\%20Publicas.pdf

Rivas, J.A. (2003). El neoinstitucionalismo y la revalorización de las instituciones. Reflexión Política, vol. 5, núm. 9, junio, 2003, pp.37-46. Universidad Autónoma de Bucaramanga Bucaramanga, Colombia.

Sánchez-Navarro, J.L., Arcas-Lario, N. \& Hernández-Espallardo, M. (2019): “Antecedentes del oportunismo en las cooperativas agroalimentarias", CIRIEC-España, Revista de Economía Pública, Social y Cooperativa, 97, 111-136. DOI: 10.7203/CIRIEC-E.97.13282

Schwettmann, J. (s/f). The Role of Cooperatives in Achieving the Sustainable Development Goals - the economic dimension https://ccr.ica.coop/sites/ccr.ica.coop/files/attachments/1.1\%20Jurgen\%20Sch wettmann.pdf 